\title{
Neuropsychiatric symptoms and the diagnostic stability of mild cognitive impairment
}

\author{
Michael A. Sugarman ${ }^{1,2}$, Michael L. Alosco ${ }^{1,3}$, Yorghos Tripodis ${ }^{1,4}$, Eric G. Steinberg ${ }^{1}$, and \\ Robert A. Stern ${ }^{1,3,5,6,{ }^{*}}$ \\ ${ }^{1}$ Boston University Alzheimer's Disease Center \\ 2Department of Psychology, Bedford Veterans Affairs Medical Center \\ ${ }^{3}$ Department of Neurology, Boston University School of Medicine \\ ${ }^{4}$ Department of Biostatistics, Boston University School of Public Health \\ ${ }^{5}$ Department of Neurosurgery, Boston University School of Medicine \\ ${ }^{6}$ Department of Anatomy and Neurobiology, Boston University School of Medicine
}

\begin{abstract}
Background: Mild cognitive impairment (MCI) is an intermediate diagnosis between normal cognition (NC) and dementia, including Alzheimer's disease (AD) dementia. However, MCI is heterogeneous; many individuals subsequently revert to $\mathrm{NC}$ while others remain stable at $\mathrm{MCI}$ for several years. Identifying factors associated with this diagnostic instability could assist in defining clinical populations and determining cognitive prognoses.
\end{abstract}

Objective: The current study examined whether neuropsychiatric symptoms could partially account for the temporal instability in cognitive diagnoses.

Method: The sample included 6763 participants from the National Alzheimer's Coordinating Center Uniform Data Set. All participants had NC at baseline, completed at least two follow-up visits (mean duration: 5.5 years), and had no recent neurological conditions. Generalized linear models estimated by generalized estimating equations examined associations between changes in cognitive diagnoses and symptoms on the Neuropsychiatric Inventory Questionnaire (NPI-Q) and Geriatric Depression Scale (GDS-15).

Results: 1121 participants converted from NC to MCI; 324 reverted back to NC and 242 progressed to AD dementia. Higher symptoms on the GDS-15 and circumscribed symptom domains on the NPI-Q were associated with conversion from NC to $\mathrm{MCI}$ and a decreased likelihood of reversion from MCI to NC. Individuals with higher symptoms on NPI-Q Hyperactivity and Mood items were more likely to progress to AD dementia.

Discussion: The temporal instability of MCI can be partially explained by neuropsychiatric symptoms. Individuals with higher levels of specific symptoms are more likely to progress to AD

*Corresponding author: bobstern@bu.edu, Boston University Alzheimer's Disease and CTE Center, 72 East Concord Street, Suite B7800, Boston, MA 02118, USA. 
dementia and less likely to revert to NC. Identification and treatment of these symptoms might support cognitive functioning in older adults.

\section{Keywords}

Mild cognitive impairment; Alzheimer's disease; Neuropsychiatric symptoms; Depression; Antidepressants

\section{Introduction}

The clinical continuum of Alzheimer's disease (AD) involves the progression from normal cognition (NC) to mild cognitive impairment (MCI), and then to AD dementia. A diagnostic progression from $\mathrm{MCI}$ to $\mathrm{AD}$ dementia occurs once impairments in activities of daily living begin. $\mathrm{MCI}$, therefore, represents the stage along the clinical $\mathrm{AD}$ continuum where there is objective evidence of cognitive impairment, but without functional decline [1]. Indeed, individuals diagnosed with $\mathrm{MCI}$ in the clinical setting are more likely to be subsequently diagnosed with $\mathrm{AD}$ dementia, with an estimated annual progression rate of $8 \%$ in specialty clinics for individuals with memory disorders and $7 \%$ in non-specialist community clinics treating the general population [2]. As such, MCI functions as an intermediate diagnosis for identifying individuals who are most likely to experience further clinical decline and who might benefit from interventions such as cognitive rehabilitation [3] and/or pharmacological treatment [4].

However, some individuals with MCI experience cognitive improvement after being diagnosed with MCI and revert back to NC, with a one-year reversion rate of about $14 \%$ in specialty memory clinics and up to $31 \%$ in community clinics [5]. Further, a meta-analysis found that in studies with an average follow-up rate of 4.6 years, the progression rates from MCI to AD dementia were $34 \%$ and $29 \%$ in specialty memory clinics and community clinics, respectively [2]. Thus, while MCI may be a valuable prodromal diagnosis, it is marked by temporal instability and an uncertain time course. This is problematic because an MCI diagnosis is often used as part of the entry criteria for clinical trials, and its diagnostic unreliability may disrupt the development of interventions for $\mathrm{AD}$. That is, individuals with MCI who subsequently revert to NC may not have underlying AD pathology, and their inclusion in research studies may increase the heterogeneity of the intended population and potentially contribute to failures in clinical trials (e.g., [6]). Examination of clinical factors associated with changes in diagnostic status is critical to improve the ability to identify who is most likely to convert to $\mathrm{MCI}$, revert to $\mathrm{NC}$, and progress to $\mathrm{AD}$ dementia and to develop and implement appropriate interventions.

Extant research has attempted to identify the clinical modifiers of both conversion to MCI and reversion from MCI to NC. Variables associated with conversion from NC to MCI include possession of the Apolipoprotein $\varepsilon 4(A P O E \varepsilon 4)$ allele, depression, type II diabetes, tobacco use, physical inactivity, and poor diet [7]. Factors that increase the probability of reverting from $\mathrm{MCI}$ to $\mathrm{NC}$ include younger age, female gender, lack of subjective memory complaints, an absence of the $A P O E \mathcal{E} 4$ allele, lower Clinical Dementia Rating (CDR) Scale-Sum of Boxes, functional independence, higher visual acuity, engagement in 
cognitively stimulating activities, and higher scores on measures of memory, executive functioning, and language [8-11]. Notably, individuals with non-amnestic MCI are more likely to revert than amnestic MCI [9].

Neuropsychiatric symptoms, such as depression, anxiety, irritability, apathy, and sleep disturbance, may also contribute to the temporal instability of MCI. Higher levels of neuropsychiatric symptoms in individuals with $\mathrm{NC}$ are associated with a higher incidence of subsequent MCI [12], and patients with MCI typically show higher rates of these symptoms than cognitively intact controls [13]. Among individuals with MCI, the presence of neuropsychiatric symptoms, particularly apathy, increases the probability of a subsequent clinical diagnosis of AD dementia [14-17]. Similarly, a diagnosis of depression is also a risk factor for subsequent diagnoses of MCI and AD dementia [18]. Symptoms of both depression [19] and anxiety [20] are associated with poorer cognitive functioning that may make it more likely for an individual to receive a diagnosis of MCI. Additionally, neuropsychiatric symptoms can often represent the earliest behavioral manifestations of underlying dementia pathology [16]. These clinical associations are supported by neurobiological research linking these symptoms to biomarkers for AD pathology. Acute stress is associated with dysregulation of the hypothalamic-pituitary-adrenal (HPA) axis, which can in turn trigger hippocampal cell death via glutamate excitotoxicity [21] and increase production of hippocampal Aß [22]. Indeed, non-demented adults with depression typically have greater amounts of $A ß$ accumulation [23], lower levels CSF Aß peptides [24], and smaller hippocampi; a meta-analysis of volumetric MRI studies found reductions of $8 \%$ and $10 \%$ in left and right hippocampi, respectively [25]. Thus, there is a clear association between neuropsychiatric symptoms and the presence of underlying AD neuropathology; this relationship may result in higher frequencies of clinical diagnoses of MCI and AD dementia amongst individuals with higher levels of neuropsychiatric symptoms.

Based on the above, it is also plausible that a reduction in neuropsychiatric symptoms may correspond with cognitive improvement among individuals with MCI and in turn be associated with a reversion to NC. Successful treatment of depression in older adults with cognitive impairment has been associated with improvements on neuropsychological testing [26] and potentially neuroprotective effects. For example, antidepressant treatment has been associated with increased hippocampal neurogenesis in rats [27] and in humans. Frodl et al. [28] observed in a sample of middle-aged adults with major depression (mean age $=45$ years) that continuous antidepressant use over a three-year period was associated with an increase in left (but not right) hippocampal volume. No significant changes in hippocampal volume were observed in their non-depressed control group or in patients with depression who discontinued antidepressant use. This study did not provide information on whether participants were engaged in other types of treatment for depression (e.g., psychotherapy, exercise intervention) and thus it is unclear whether the hippocampal growth is specific to antidepressant treatment or a more general effect associated with the remission of depression. Alternatively, an improvement in neuropsychiatric symptoms could accompany an improvement of an underlying condition that could be also be causing cognitive impairment (e.g., hypoglycemia, metabolic abnormalities, etc.). 
Although previous research has established associations between neuropsychiatric symptoms and progression to MCI and dementia, less research has examined the associations between neuropsychiatric symptoms and reversion from MCI to NC. Koepsell and Monsell [9] observed that individuals diagnosed with MCI and a primary suspected etiology of depression had a higher probability of reverting to a diagnosis of either NC or "cognitively impaired, not MCI" at one-year follow-up (21\%) compared to the sample as a whole (16\%). Pandya et al. [11] examined circumscribed informant-reported symptoms on the Neuropsychiatric Inventory Questionnaire (anxiety, depression, and apathy) and found that fewer symptoms amongst individuals with MCI at baseline were associated with an increased probability of reversion to NC. However, both these studies utilized a prediction model and did not analyze the level of symptoms at the time point where participants reverted. Such an analysis could determine whether reversion to NC is characterized by a low level of neuropsychiatric symptoms and/or an improvement in symptoms after receiving a diagnosis of MCI.

The objective of the current study was to examine associations between neuropsychiatric symptoms and the temporal stability of MCI using the National Alzheimer's Coordinating Center (NACC) longitudinal Uniform Data Set (UDS). We examined the relationships between neuropsychiatric symptoms at each time point (as well as changes from the previous evaluation) and 1) conversion from $\mathrm{NC}$ to $\mathrm{MCI}, 2$ ) reversion from $\mathrm{MCI}$ to $\mathrm{NC}$, and 3) progression from MCI to AD dementia. We hypothesized that individuals with higher levels of neuropsychiatric symptoms would be more likely to convert from NC to MCI and to progress from MCI to AD dementia, and individuals with lower levels of symptoms would be more likely to revert to NC. Specifically, we hypothesized that affective symptoms (e.g., depressed mood, apathy, anxiety, etc.) would be most likely to mirror changes in cognitive diagnoses, given their relationships with cognitive functioning. Further, amongst individuals with MCI, pharmacological treatment for neuropsychiatric symptoms would be associated with an increased likelihood of reversion to NC.

\section{Method}

\section{Participants}

The current sample included 6763 participants from the NACC-UDS who were all diagnosed with NC at study entry. The NACC was established in 1999 by the National Institute on Aging (NIA) to facilitate AD research. The UDS is a publicly accessible longitudinal database gathered from 31 Alzheimer's Disease Centers (ADCs) across the United States. Beginning in 2005, each ADC began to contribute data from standardized annual evaluations pertaining to participants' cognitive, behavioral, and functional abilities. For a more comprehensive description of the NACC-UDS, please refer to Weintraub et al. [29], Beekly et al. [30,31], and Morris et al. [32]. All research using the NACC database is approved by the University of Washington Institutional Review Board. Informed consent from all participants was obtained at their respective ADCs.

NACC granted access to these data following a formal request (proposal ID 809) and provided data from the UDS collected between September 2005 and September 2016. The sample was restricted to participants with a diagnosis of NC at baseline and had a total of at 
least three visits (i.e., two or more follow-up visits; initial $N=7919$ ) in order to facilitate derivation of a sample with multiple diagnostic conversions. Several exclusion criteria were applied to increase generalizability to $\mathrm{AD}$ and limit confounds for changes in diagnostic status over time. Participants were excluded if they were younger than age 50, had a neurological diagnosis other than suspected AD (i.e., Parkinson's disease, frontotemporal dementia, corticobasal degeneration, vascular dementia, a recent traumatic brain injury with an extended loss of consciousness, or recent history of stroke or seizure), and individuals who were prescribed lithium and/or antipsychotic medications, given the relationships between these medications and cognitive impairment. The sample size for all analyses was reduced to 6763 after applying these exclusion criteria. Additionally, previous examinations of NACC data (e.g., [9]) observed that different ADCs inconsistently utilize the "cognitively impaired, not MCI" designation; thus, study visits including this designation were excluded from analyses. Other study visits from participants who received this diagnosis at least once were still eligible for inclusion in the analyses.

All study visits occurred between 2005 and 2016, and participants had a total of between 3 and 12 visits (mean $=5.71, \mathrm{SD}=2.31$ ). Study visits occurred on an annual basis. The obtained dataset included variables pertaining to participant demographics, medications, medical and psychiatric diagnoses, and cognitive diagnosis (i.e., NC; cognitively impaired, not MCI; MCI; dementia). Diagnoses were determined through multidisciplinary consensus conferences (68.0\% of study visits), a single clinician's diagnosis (30.6\%), or another means such as an informal consensus between multiple clinicians (1.4\%). MCI diagnoses were based on the criteria defined by Winblad et al. [33], and AD dementia was diagnosed based on criteria from the National Institute of Neurological and Communicative Disorders and Stroke/Alzheimer's Disease and Related Disorders Association [34].

The two primary neuropsychiatric variables in analyses were the Neuropsychiatric Inventory Questionnaire (NPI-Q) and the Geriatric Depression Scale 15-item short form (GDS-15). The NPI-Q [35] asks study partners to evaluate the presence or absence of 12 neuropsychiatric symptoms that may be present in dementia over the past month. Each symptom is evaluated for the presence and severity of the symptom on a scale from 0 (absent) to 3 (severe); frequency values were not available for analysis in the current dataset. Previous analyses of the NPI-Q have demonstrated the value of separating the items into different symptom clusters rather than a total summary score $[12,17,36]$. We adapted the results from a factor analysis from a sample of outpatients with dementia, $73 \%$ of whom were diagnosed with AD dementia [36]. Their results indicated that neuropsychiatric symptoms clustered into three main categories of hyperactivity (agitation, euphoria, irritability, disinhibition, and aberrant motor behavior), psychotic symptoms (delusions, hallucinations), and mood (depression, apathy, nighttime behavior disturbances, and appetite/eating abnormalities), accounting for the presence, severity, and frequency of these symptoms. Anxiety did not load cleanly onto any of the three factors and was interpreted as a separate symptom. Thus, the NPI-Q was analyzed as four separate variables (i.e., hyperactivity, psychosis, mood, and anxiety). Each of the factor scores were calculated by summing the severity ratings for the respective items. The GDS-15 [37] asks participants to 
answer 15 yes/no questions pertaining to symptoms of depression. Higher scores on this measure represent a greater level of depressive symptoms. Neuropsychiatric symptoms were examined both as the level of symptoms at the time of diagnostic changes (i.e., the three critical events described below) and as the change in symptom level from the previous study visit by subtracting the value from the previous measurement. Thus, for each follow-up visit, a level of current symptoms and an estimate of symptom trajectory was available for each participant.

\section{Statistical Analyses}

All study hypotheses were evaluated using generalized linear models (GLM) estimated by generalized estimating equations (GEE) [38], which is an appropriate estimation method for correlated data such as longitudinal analyses. Analyses were conducted using the "geepack" package [39] for R [40], using an autoregressive (AR1) correlation structure. Binomial logistic regression models estimated by GEE examined the three binary outcome variables, which were the critical events of 1) conversion from NC to MCI, 2) reversion from MCI to $\mathrm{NC}$, and 3) progression from MCI to AD dementia. If a single participant had multiple events of the same type (e.g., converting to $\mathrm{MCI}$, reverting to $\mathrm{NC}$, and then reverting again to $\mathrm{MCI}$ ) only the first observed event was included in analyses.

In all models, the outcome was coded as a binary variable detailing the changes in diagnostic status from the previous study visit, with " 1 " representing one of the three critical events and " 0 " representing stable diagnoses across visits. Consequently, the initial study visit was coded as missing and was excluded from all analyses. The study visits in the models included visits where participants were stable on the baseline condition as well as visits with the critical event. For example, models examining conversion to MCI included visits where participants were stable at $\mathrm{NC}$ as well as the first visit where they were diagnosed with MCI, if this occurred. Models examining reversion to NC included study visits with stable diagnoses of MCI and visits where reversion occurred. Thus, the coefficients in the models represent the associations between the variable and the critical event compared with remaining diagnostically stable. The natural exponents of the coefficients $(\operatorname{Exp}[\mathrm{B}])$ can be interpreted as the percentage change in the probability of the critical event for each unit increase in the predictor. GEE allows for covariance between variables and logistic regression does not make assumptions regarding the distribution of variables; thus, no transformations were applied to any variables. Linearity between continuous independent variables and the log odds of the dependent variables was confirmed using the Box-Tidwell test [41]. The time (in years) since baseline and the demographics of age (at baseline), sex, education, race, and ethnicity were included as covariates in all models. Preliminary data analyses revealed that race and ethnicity were not significant predictors of any of the three critical events and these variables were not included in the final models. For analyses examining reversion to $\mathrm{NC}$ or progression to $\mathrm{AD}$, the subtype of $\mathrm{MCI}$ was also included as a baseline predictor. Subtypes were dichotomized into amnestic vs. non-amnestic MCI, as a previous analysis [9] observed that individuals with non-amnestic MCI were more likely to revert to NC. 
The relationships between neuropsychiatric symptoms and diagnostic change were examined two ways: 1) by examining the associations between the GDS-15 total score and the four NPI-Q index scores at each study visit and diagnostic status, and 2) examining the association between changes in neuropsychiatric symptoms between study visits as a predictor of diagnostic change; positive values represent worsening symptomatology since the previous visit. In this way, we examined the relationships between both the total level of symptoms as well as recent exacerbations or improvements in symptoms and the three critical events. Additionally, we examined whether prescriptions for antidepressants, anticonvulsants/mood stabilizers, benzodiazepines, and/or cognition-enhancing medications (see Table 1 for a list of medications examined) were significantly related to the three critical events, as well as whether a new prescription for individuals with MCI was associated with reversion to $\mathrm{NC}$.

\section{Results}

\section{Demographics and cognitive trajectories}

Table 2 displays the demographic characteristics for the entire sample as well as participants who experienced any of the three critical events. In total, 1121 of the 6763 participants converted from NC to MCI; 860 of these participants were classified as amnestic MCI (single or multiple domain) and the remaining 261 had non-amnestic MCI. Three hundred twenty-four participants reverted from MCI to NC at a subsequent study visit, and 242 progressed from MCI to dementia with a presumptive etiology of possible or probable AD. Figure 1 provides a flowchart detailing the number of individuals which each cognitive diagnosis at all 12 study visits, along with the number of critical events at each visit.

\section{Relationship between neuropsychiatric symptoms and change in diagnostic status}

Table 3 displays the results from five logistic GEE models predicting the likelihood of conversion from NC to MCI. Model 1A displays the baseline covariates that were included in all subsequent models, demonstrating that participants who converted were significantly more likely to be older, male, and have a lower level of education ( $p$-values $<.001)$. Time since baseline was not a significant predictor of cognitive change. Models 1B demonstrates that a higher total score on the GDS-15 was significantly related to conversion to MCI (19\% increased probability per point). With regard to the NPI-Q, only the scores for Hyperactivity, Mood, and Anxiety symptoms were significantly related to conversion $(p$-values $<.001)$. Models 1D and 1E indicate that exacerbations of symptoms between study visits on the GDS-15 and the Hyperactivity and Mood domains of the NPI-Q were also significantly related to conversion to $\mathrm{MCI}$.

Table 4 displays the relationship between neuropsychiatric symptoms and the odds of reversion from MCI to NC. Model 2A displays the baseline covariates, showing that individuals who reverted tended to be younger, more likely to revert closer in time to the initial study visit, and have a classification of non-amnestic MCI $(p$-values $<.01)$. Lower total scores on the GDS-15 (Model 2B) were associated with a significantly higher probability of reversion ( $p$-value $=.003)$. A reduction of depressive symptoms on the GDS-15 between study visits indicated an increased likelihood of reversion (7\% per point 
decrease; Model 2D). Individuals who had lower informant-reported Mood and Anxiety symptoms on the NPI-Q were also more likely to revert (Model 2C). However, the change in these symptom levels between study visits was not significantly related to reversion (Model 2E).

Table 5 displays the relationship between neuropsychiatric symptoms and progression from MCI to AD. The baseline covariates (Model 3A) show that participants who progressed to dementia tended to be older at baseline, progressed at a later study visit, and have a diagnosis of amnestic MCI ( $p$-values < .01). The GDS-15 was not significantly related to progression to $\mathrm{AD}$ (Models 3B and 3D). However, a higher total number of symptoms on the Hyperactivity and Mood symptoms on the NPI-Q (Model 3C) as well as an increase in these symptom domains since the previous study visit (Model 3E) both indicated an increased probability of progressing to dementia.

\section{Relationships between medications and changes in cognitive status}

Table 6 displays the relationships between conversion to MCI and prescriptions for the four classes of psychotropic medications detailed in Table 1. In each model, the same covariates from the previous analyses were included (Model 1A). Those taking an antidepressant were $58 \%$ more likely to convert to MCI ( $p$-value < .001; Model 4A). However, this relationship appeared to be mediated by neuropsychiatric symptoms, and when the GDS-15 and the symptom domains from the NPI-Q that were significantly related to conversion (i.e., Hyperactivity, Mood, and Anxiety) were also included as predictors (Model 4B), antidepressants were no longer significantly associated with conversion $(p$-value $=.53)$. Those with a prescription for anticonvulsants were $70 \%$ more likely to convert to $\mathrm{MCI}$ ( $p$ value $=.04$; Model $4 \mathrm{C}$ ). This relationship was partially mediated by the simultaneous inclusion of the neuropsychiatric symptoms significantly related to conversion (Model 4D); after accounting for these symptoms, anticonvulsants were no longer a statistically significant predictor of reversion ( $p$-value $=.13$ ). Benzodiazepines (Model 4E) were not significantly related to conversion to MCI. Cognition-enhancing medications were associated with an exceptionally high probability of conversion to MCI (Model 4F), but it is important to note that only 12 participants in the entire sample were prescribed one of these drugs at the time of conversion. This relationship remained significant after including neuropsychiatric symptoms as simultaneous predictors (Model 4G).

Table 7 displays the relationships between the medication classes and reversion from MCI to NC. The predictors in each model were added to the baseline covariates in Model 2A. Having a prescription for an antidepressant was not significantly related to the likelihood of reversion (Model 5A), nor was receiving a new antidepressant prescription between study visits after receiving a diagnosis of MCI (Model 5B). A non-significant negative relationship was observed between benzodiazepine prescriptions and reversion (Model 5C). Taking a cognition-enhancing medication was associated with a decreased likelihood of reverting to NC (Model 5D), and receiving a new prescription for one of these drugs following a diagnosis of MCI was also associated with a reduced probability of reversion (Model 5E). With regard to anticonvulsants, an insufficient number of participants were taking an anticonvulsant to provide a reliable analysis of the relationship between these medications 
and reversion; only four participants who reverted and 18 participants with a stable diagnosis of MCI had a prescription for one of these medications.

Finally, Table 8 displays the relationships between the four medication classes and the probability for progressing from a diagnosis of MCI to AD dementia. Participants who were taking an antidepressant were $100 \%$ more likely to receive a diagnosis of dementia (Model 6A); after accounting for the NPI-Q symptoms that were significantly related to progression to $\mathrm{AD}$ (i.e., Hyperactivity and Mood), the relationship between antidepressants remained significant but was of a smaller magnitude (62\% increase in probability; Model 6B). Benzodiazepine prescriptions were not significantly associated with progressing to $\mathrm{AD}$ (Model 6C). With regard to cognition-enhancing medications, these participants were far more likely to progress to dementia (Model 6D), and this relationship remained significant after accounting for neuropsychiatric symptoms (Model 6E). When the analysis was restricted to individuals who received a new prescription after converting to $\mathrm{MCI}$, a similar relationship emerged (Model 6F) that also remained significant after accounting for neuropsychiatric symptoms (Model 6G).

\section{Discussion}

In this longitudinal study of 6763 participants from the NACC-UDS, neuropsychiatric symptoms significantly contributed to temporal instability in cognitive diagnoses.

Specifically, higher levels of self- and informant-reported symptoms increased the likelihood of conversion from NC to MCI, with the exception of psychotic symptoms on the NPI-Q. For individuals with MCI, those with lower levels of self-reported depression and fewer informant-reported mood and anxiety symptoms were more likely to revert back to NC. Further, an improvement of symptoms on the GDS-15 indicated a higher likelihood of reverting to $\mathrm{NC}$, indicating that successful reduction of depressive symptoms may accompany improvements in cognitive functioning. Progression from MCI to AD dementia was characterized by more severe mood and hyperactivity symptoms on the NPI-Q; selfreported depressive symptoms on the GDS-15 were not significantly related to risk for progression from MCI to AD dementia. Overall, the results indicate that trajectory of neuropsychiatric symptoms may accompany some corresponding changes in cognitive diagnoses. This relationship may reflect how more severe mood and behavioral dysfunction can adversely impact cognitive functioning and/or demonstrate how these neuropsychiatric symptoms are part of the core clinical presentation of AD. Individuals with MCI and selfreported symptoms of depression may have potentially reversible cognitive impairment. High levels of informant-reported neuropsychiatric symptoms (e.g., hyperactivity and mood symptoms) may more likely reflect the progression of an underlying AD dementia.

A crucial finding from analyses is that individuals who experience a successful reduction in self-reported symptoms of depression (i.e., on the GDS-15) may have a higher probability of reverting from MCI to NC. Thus, individuals with depression and MCI might benefit from treatment for depression. However, having a prescription for an antidepressant did not impact the odds of reverting to NC. An important limitation is that this finding is based on whether participants had a prescription at the time of the study visit and does not account for whether they were actually taking the medication as prescribed; antidepressant non- 
adherence has been estimated as high as 73\% [42]. An additional limitation is that we did not have information as to the reasons for the prescriptions; for some patients, they may have been prescribed to assist with challenging behaviors that could be due to AD pathology (e.g., sundowning) rather than for treatment of depression. In any case, combination therapy including pharmacotherapy and psychotherapy has been demonstrated to be superior to pharmacotherapy alone $[43,44]$. Additionally, exercise is not only an effective intervention for treating depression [45] but it has also been shown to increase hippocampal volume and improve aspects of cognition in older adults [46] and as such, it may be indicated for individuals with MCI. Thus, there are two potential mechanisms by which an improvement in depressive symptoms may improve cognition: 1) reducing symptoms that directly impact cognition (e.g., rumination, worry, sleep disturbance) and 2) limiting or compensating for neurobiological changes that may be related to a dementia process (e.g., delaying or reversing hippocampal atrophy). However, an additional explanation for the findings is that some participants may have had a resolution of a condition that was simultaneously causing mood disruption and cognitive impairment (e.g., hypoglycemia, metabolic disturbances, chronic pain, etc.). A future research direction to further explore these possibilities could include formal clinical trials targeted at patients with MCI and depression to determine whether treatment for depression leads to improvements in cognitive functioning.

The relationship between the resolution of depression and reversion to $\mathrm{NC}$ also indicates that individuals with depression and MCI (particularly non-amnestic MCI) may be less likely to be developing $\mathrm{AD}$ dementia. As such, these individuals may confound the results of clinical trials intended to develop interventions for patients with MCI to delay or prevent AD dementia. Thus, the results from the current study support clinical trial practices of excluding MCI patients with depression [6]. Although these participants may not be the best candidates for inclusion in clinical trials, it may be inappropriate to call MCI a misdiagnosis for patients with depression. The depression may be present in addition to or as a result of legitimate AD pathology. Further, individuals who revert from MCI to NC appear to be more likely to subsequently receive a diagnosis of MCI and/or dementia compared to individuals with NC who had never received a diagnosis of MCI [10]. Indeed, within our sample, 95 out of $324(29.3 \%)$ individuals who reverted from MCI to NC eventually reconverted to MCI at a subsequent study visit. Thus, a diagnosis of MCI at any time point appears to have prognostic value for future cognitive status, and it has been argued that an MCI should be considered a heterogeneous clinical diagnosis indicating a high risk for subsequent progression to dementia rather than be diagnostic of any specific disease pathology [47].

Examination of the four symptom domains on the NPI-Q revealed that certain domains had stronger relationships with changes in cognitive diagnoses than others. All domains other than psychosis were significantly associated with conversion to MCI. Lower symptoms on only the mood (i.e., depression, apathy, nighttime behaviors, and appetite disturbance) and anxiety factors were associated with a higher probability of reversion to NC, consistent with previous research [11], whereas higher symptoms on the mood and hyperactivity (i.e., agitation, euphoria, irritability, disinhibition, and aberrant motor behavior) were associated with progression to AD dementia. These findings indicate that individuals with MCI with less anxiety and who are exhibiting fewer of the mood symptoms that characterize AD may be good candidates to revert to NC. Amongst individuals with MCI, higher mood and 
hyperactivity symptoms may likely represent neurobehavioral manifestations of underlying AD pathology; these individuals are more likely to progress to dementia. The relationship with mood symptoms and dementia is consistent with previous research indicating that apathy may represent an early clinical feature of AD [14,15]. In fact, given how neuropsychiatric symptoms can indicate underlying dementia pathology, some have argued that the presence of neuropsychiatric symptoms even without concurrent cognitive impairment should warrant a separate diagnostic label (known as "Mild Behavioral Impairment") to identify individuals at a higher risk for dementia [16].

There were no significant relationships between psychotic symptoms (i.e., hallucinations and delusions) and changes in cognitive diagnoses; however, it is notable that the overall prevalence of these symptoms was exceptionally low in the sample. The mean symptom level for individuals converting from NC to MCI was 0.04 out of 6; for participants who progressed to $\mathrm{AD}$ dementia, the mean was 0.19 at the time of the diagnosis. Thus, the prevalence of psychotic features may not be a sensitive variable for the trajectory of cognitive functioning amongst individuals with $\mathrm{NC}$ or $\mathrm{MCI}$. This finding is consistent with previous research indicating that psychotic features are more likely to be present later in the disease course of $\mathrm{AD}$, when individuals have moderate-to-severe dementia [48].

An interesting discrepancy emerged between the GDS-15, a self-report measure, and the NPI-Q, an informant-reported measure. Higher mood symptoms on the NPI-Q were associated with progression from MCI to AD dementia; however, there was not a significant relationship between the GDS-15 and progression to dementia. This may represent worsening anosognosia in individuals with AD dementia [49], including the ability to accurately self-report psychiatric symptoms [50]. Bruce et al. [51] found that amongst patients with MCI, those with higher levels of self-reported depression paradoxically performed better on measures of memory abilities, reflecting how these individuals may have higher insight about their psychiatric status. Thus, the current findings are consistent with the literature by indicating that individuals with MCI and self-reported depression may be strong candidates for reversion to NC [9].

An analysis of the impact of medication prescriptions did not indicate that prescriptions for antidepressants or cognition-enhancing medications could increase the probability of reversion from MCI to NC. In fact, individuals with these prescriptions appeared to be more likely to experience to progress within the clinical continuum of $\mathrm{AD}$ (i.e., from $\mathrm{NC}$ to $\mathrm{MCI}$ to dementia). However, there are a number of limitations that temper the interpretation of these findings. As mentioned above, the database solely listed participant prescriptions at the time of the study visit without further information such as dosage or adherence. Crucially, there was not an indicated reason why the medication was prescribed, and as such it is not possible to deduce a direct relationship between medications and outcome due to the potential confound of indication. For example, many of the medications were likely prescribed to help manage symptoms indicative of underlying AD pathology (e.g., memory impairment, apathy, agitation), meaning that these individuals were already at a higher risk for cognitive deterioration; a prescription medication would be unlikely to dramatically alter the disease course. Additionally, participants receiving the medications may have had additional factors that also placed them at higher risk for dementia, such as medical 
comorbidities and stroke risk factors. Thus, there is insufficient evidence from these analyses to determine whether these medications are independent risk factors for cognitive decline.

A potential criticism of the analyses could be that we excluded individuals who had a prescription for lithium or an antipsychotic medication due to their impact on cognitive functioning. Notably, after applying all other exclusion criteria, only 9 individuals were excluded because of lithium and 75 were excluded because of antipsychotics. The overall incidence of progression from MCI to AD dementia in these individuals was 19.0\% (16/84), which was significantly higher than the rate of $3.6 \%(242 / 6763)$ in the analyzed sample $\left(\chi^{2}\right.$ $=50.6, p$-value $<.001)$. Although it certainly cannot be determined whether the drugs caused the higher rates of cognitive impairment or whether the drugs were prescribed to treat symptoms potentially related to AD pathology, we still believe that these exclusion criteria were appropriate. Although benzodiazepines have also been associated with cognitive impairment [52], having a prescription in our sample was not significantly associated with changes in cognitive diagnoses (Tables 4-6). Further, the overall incidence of progression from MCI to AD dementia in individuals with a prescription was $4.6 \%(28 / 607)$, which was not significantly higher than the rate of $3.5 \%(214 / 6156)$ in the rest of the sample $\left(\chi^{2}=1.75\right.$, $p$-value $=.186)$.

Additional limitations of the current study include the observational nature of the dataset, which limits causal implications. An additional limitation is the classification of individuals into the discrete categories of NC, MCI, and AD for the outcome variables. The use of continuous outcome measures such as neuropsychological testing and/or activities of daily living rating scales may accounted for some of the unexplained variance in the analyses. However, we chose to use these categories as the outcome to facilitate clinical and research generalizability, as these labels are heavily utilized in both practices and are often used as part of the entry criteria for clinical trials. A further limitation is the heterogeneity of the NACC-UDS database, which pools data from 34 separate research centers with volunteer participants. Although each center collects standardized data as part of their annual evaluations, they may have separate inclusion/exclusion criteria. Additionally, even though the use of formal diagnostic criteria for cognitive diagnoses should partially counteract this heterogeneity, there appears to be systematic differences between sites with regard to the use of the "cognitively impaired, not MCI" diagnosis [9]. Thus, it is unclear to what extent these findings may generalize to other settings; in any situation, it is recommended that clinicians attempt to use formal criteria when making diagnoses of MCI and/or dementia. Even with the use of formal criteria, there is a potential for diagnostic misclassification due to the imperfect reliability of neuropsychological tests and clinical raters, which may have contributed to unexplained variance in analyses. Another limitation is that information pertaining to APOE genotype was not included in analyses, which could have reduced unexplained variance. Notably, we did obtain this information for participants in the sample from the NACC Genetics Dataset. However, APOE genotype information was missing for 871 participants (13\% of the total available sample). Upon further investigation, individuals with missing genotype data were on average older, had lower education, and were more likely to be of a minority ethnic background. Given that the inclusion of this variable would reduce the sample size considerably and how the missing data appeared to be systematic in nature, we opted to exclude this variable from analyses. A final limitation is that there was 
no information available about whether participants were engaged in other treatments for psychiatric conditions such as psychotherapy or exercise that could potentially account for changes in neuropsychiatric symptoms over time.

In conclusion, the results indicate that monitoring for neuropsychiatric symptoms can provide valuable information about which individuals are most likely convert to MCI, revert to $\mathrm{NC}$, and progress to AD dementia. Successfully identifying and providing effective treatment for these symptoms (particularly self-reported depression) may potentially improve or maintain cognitive functioning in older adults.

\section{Acknowledgements}

The NACC database is funded by NIA/NIH Grant U01 AG016976. NACC data are contributed by the NIA-funded ADCs: P30 AG019610 (PI Eric Reiman, MD), P30 AG013846 (PI Neil Kowall, MD), P50 AG008702 (PI Scott Small, MD), P50 AG025688 (PI Allan Levey, MD, PhD), P30 AG010133 (PI Andrew Saykin, PsyD), P50 AG005146 (PI Marilyn Albert, PhD), P50 AG005134 (PI Bradley Hyman, MD, PhD), P50 AG016574 (PI Ronald Petersen, MD, PhD), P50 AG005138 (PI Mary Sano, PhD), P30 AG008051 (PI Steven Ferris, PhD), P30 AG013854 (PI M. Marsel Mesulam, MD), P30 AG008017 (PI Jeffrey Kaye, MD), P30 AG010161 (PI David Bennett, MD), P30 AG010129 (PI Charles DeCarli, MD), P50 AG016573 (PI Frank LaFerla, PhD), P50 AG016570 (PI David Teplow, PhD), P50 AG005131 (PI Douglas Galasko, MD), P50 AG023501 (PI Bruce Miller, MD), P30 AG035982 (PI Russell Swerdlow, MD), P30 AG028383 (PI Linda Van Eldik, PhD), P30 AG010124 (PI John Trojanowski, MD, PhD), P50 AG005133 (PI Oscar Lopez, MD), P50 AG005142 (PI Helena Chui, MD), P30 AG012300 (PI Roger Rosenberg, MD), P50 AG005136 (PI Thomas Montine, MD, PhD), P50 AG033514 (PI Sanjay Asthana, MD, FRCP), and P50 AG005681 (PI John Morris, MD).

\section{References}

[1]. Petersen RC, Negash S (2008) Mild cognitive impairment: an overview. CNS Spectr 13: 45-53.

[2]. Mitchell AJ, Shiri-Feshki M (2009) Rate of progression of mild cognitive impairment to dementia--meta-analysis of 41 robust inception cohort studies. Acta Psychiatr Scand 119: 252265. 10.1111/j.1600-0447.2008.01326.x. [PubMed: 19236314]

[3]. Giebel C, Challis D (2015) Translating cognitive and everyday activity deficits into cognitive interventions in mild dementia and mild cognitive impairment. Int J Geriatr Psychiatry 30: 21 31. 10.1002/gps.4170. [PubMed: 24990546]

[4]. Karakaya T, Fusser F, Schroder J, Pantel J (2013) Pharmacological Treatment of Mild Cognitive Impairment as a Prodromal Syndrome of Alzheimer s Disease. Curr Neuropharmacol 11: 102108. 10.2174/157015913804999487. [PubMed: 23814542]

[5]. Malek-Ahmadi M (2016) Reversion From Mild Cognitive Impairment to Normal Cognition: A Meta-Analysis. Alzheimer Dis Assoc Disord. 10.1097/WAD.0000000000000145.

[6]. Feldman HH, Ferris S, Winblad B, Sfikas N, Mancione L, He Y, Tekin S, Burns A, Cummings J, del Ser T, Inzitari D, Orgogozo JM, Sauer H, Scheltens P, Scarpini E, Herrmann N, Farlow M, Potkin S, Charles HC, Fox NC, Lane R (2007) Effect of rivastigmine on delay to diagnosis of Alzheimer's disease from mild cognitive impairment: the InDDEx study. Lancet Neurol 6: 501512. 10.1016/S1474-4422(07)70109-6. [PubMed: 17509485]

[7]. Plassman BL, Williams JW Jr., Burke JR, Holsinger T, Benjamin S (2010) Systematic review: factors associated with risk for and possible prevention of cognitive decline in later life. Ann Intern Med 153: 182-193. 10.7326/0003-4819-153-3-201008030-00258. [PubMed: 20547887]

[8]. Sachdev PS, Lipnicki DM, Crawford J, Reppermund S, Kochan NA, Trollor JN, Wen W, Draper B, Slavin MJ, Kang K, Lux O, Mather KA, Brodaty H, Sydney Memory AST (2013) Factors predicting reversion from mild cognitive impairment to normal cognitive functioning: a population-based study. PLoS One 8: e59649 10.1371/journal.pone.0059649. [PubMed: 23544083]

[9]. Koepsell TD, Monsell SE (2012) Reversion from mild cognitive impairment to normal or nearnormal cognition: risk factors and prognosis. Neurology 79: 1591-1598. 10.1212/WNL. 0b013e31826e26b7. [PubMed: 23019264] 
[10]. Roberts RO, Knopman DS, Mielke MM, Cha RH, Pankratz VS, Christianson TJ, Geda YE, Boeve BF, Ivnik RJ, Tangalos EG, Rocca WA, Petersen RC (2014) Higher risk of progression to dementia in mild cognitive impairment cases who revert to normal. Neurology 82: 317-325. 10.1212/WNL.0000000000000055. [PubMed: 24353333]

[11]. Pandya SY, Lacritz LH, Weiner MF, Deschner M, Woon FL (2017) Predictors of Reversion from Mild Cognitive Impairment to Normal Cognition. Dement Geriatr Cogn Disord 43: 204-214. 10.1159/000456070. [PubMed: 28301848]

[12]. Leoutsakos JM, Forrester SN, Lyketsos CG, Smith GS (2015) Latent Classes of Neuropsychiatric Symptoms in NACC Controls and Conversion to Mild Cognitive Impairment or Dementia. J Alzheimers Dis 48: 483-493. 10.3233/JAD-150421. [PubMed: 26402012]

[13]. Hwang TJ, Masterman DL, Ortiz F, Fairbanks LA, Cummings JL (2004) Mild cognitive impairment is associated with characteristic neuropsychiatric symptoms. Alzheimer Dis Assoc Disord 18: 17-21. [PubMed: 15195459]

[14]. Teng E, Lu PH, Cummings JL (2007) Neuropsychiatric symptoms are associated with progression from mild cognitive impairment to Alzheimer's disease. Dement Geriatr Cogn Disord 24: 253-259. 10.1159/000107100. [PubMed: 17700021]

[15]. Palmer K, Di Iulio F, Varsi AE, Gianni W, Sancesario G, Caltagirone C, Spalletta G (2010) Neuropsychiatric predictors of progression from amnestic-mild cognitive impairment to Alzheimer's disease: the role of depression and apathy. J Alzheimers Dis 20: 175-183. 10.3233/ JAD-2010-1352. [PubMed: 20164594]

[16]. Ismail Z, Smith EE, Geda Y, Sultzer D, Brodaty H, Smith G, Aguera-Ortiz L, Sweet R, Miller D, Lyketsos CG, Area INSPI (2016) Neuropsychiatric symptoms as early manifestations of emergent dementia: Provisional diagnostic criteria for mild behavioral impairment. Alzheimers Dement 12: 195-202. 10.1016/j.jalz.2015.05.017. [PubMed: 26096665]

[17]. Forrester SN, Gallo JJ, Smith GS, Leoutsakos JM (2016) Patterns of Neuropsychiatric Symptoms in Mild Cognitive Impairment and Risk of Dementia. Am J Geriatr Psychiatry 24: 117-125. 10.1016/j.jagp.2015.05.007. [PubMed: 26209222]

[18]. Steenland K, Karnes C, Seals R, Carnevale C, Hermida A, Levey A (2012) Late-life depression as a risk factor for mild cognitive impairment or Alzheimer's disease in 30 US Alzheimer's disease centers. J Alzheimers Dis 31: 265-275. 10.3233/JAD-2012-111922. [PubMed: 22543846]

[19]. Snyder HR (2013) Major depressive disorder is associated with broad impairments on neuropsychological measures of executive function: a meta-analysis and review. Psychol Bull 139: 81-132. 10.1037/a0028727. [PubMed: 22642228]

[20]. Beaudreau SA, O'Hara R (2008) Late-life anxiety and cognitive impairment: a review. Am J Geriatr Psychiatry 16: 790-803. 10.1097/JGP.0b013e31817945c3. [PubMed: 18827225]

[21]. Nehls M (2016) Unified theory of Alzheimer's disease (UTAD): implications for prevention and curative therapy. J Mol Psychiatry 4: 3 10.1186/s40303-016-0018-8. [PubMed: 27429752]

[22]. Dong H, Csernansky JG (2009) Effects of stress and stress hormones on amyloid-beta protein and plaque deposition. J Alzheimers Dis 18: 459-469. 10.3233/JAD-2009-1152. [PubMed: 19584430]

[23]. Wu KY, Hsiao IT, Chen CS, Chen CH, Hsieh CJ, Wai YY, Chang CJ, Tseng HJ, Yen TC, Liu CY, Lin KJ (2014) Increased brain amyloid deposition in patients with a lifetime history of major depression: evidenced on 18F-florbetapir (AV-45/Amyvid) positron emission tomography. Eur J Nucl Med Mol Imaging 41: 714-722. 10.1007/s00259-013-2627-0. [PubMed: 24233127]

[24]. Pomara N, Bruno D, Sarreal AS, Hernando RT, Nierenberg J, Petkova E, Sidtis JJ, Wisniewski TM, Mehta PD, Pratico D, Zetterberg H, Blennow K (2012) Lower CSF amyloid beta peptides and higher F2-isoprostanes in cognitively intact elderly individuals with major depressive disorder. Am J Psychiatry 169: 523-530. 10.1176/appi.ajp.2011.11081153. [PubMed: 22764362]

[25]. Videbech P, Ravnkilde B (2004) Hippocampal volume and depression: a meta-analysis of MRI studies. Am J Psychiatry 161: 1957-1966. 10.1176/appi.ajp.161.11.1957. [PubMed: 15514393]

[26]. Butters MA, Becker JT, Nebes RD, Zmuda MD, Mulsant BH, Pollock BG, Reynolds CF 3rd, (2000) Changes in cognitive functioning following treatment of late-life depression. Am J Psychiatry 157: 1949-1954. 10.1176/appi.ajp.157.12.1949. [PubMed: 11097959] 
[27]. Malberg JE, Eisch AJ, Nestler EJ, Duman RS (2000) Chronic antidepressant treatment increases neurogenesis in adult rat hippocampus. J Neurosci 20: 9104-9110. [PubMed: 11124987]

[28]. Frodl T, Jager M, Smajstrlova I, Born C, Bottlender R, Palladino T, Reiser M, Moller HJ, Meisenzahl EM (2008) Effect of hippocampal and amygdala volumes on clinical outcomes in major depression: a 3-year prospective magnetic resonance imaging study. J Psychiatry Neurosci 33: 423-430. [PubMed: 18787661]

[29]. Weintraub S, Salmon D, Mercaldo N, Ferris S, Graff-Radford NR, Chui H, Cummings J, DeCarli C, Foster NL, Galasko D, Peskind E, Dietrich W, Beekly DL, Kukull WA, Morris JC (2009) The Alzheimer's Disease Centers' Uniform Data Set (UDS): the neuropsychologic test battery. Alzheimer Dis Assoc Disord 23: 91-101. 10.1097/WAD.0b013e318191c7dd. [PubMed: 19474567]

[30]. Beekly DL, Ramos EM, van Belle G, Deitrich W, Clark AD, Jacka ME, Kukull WA, Centers NIAsD (2004) The National Alzheimer's Coordinating Center (NACC) Database: an Alzheimer disease database. Alzheimer Dis Assoc Disord 18: 270-277. [PubMed: 15592144]

[31]. Beekly DL, Ramos EM, Lee WW, Deitrich WD, Jacka ME, Wu J, Hubbard JL, Koepsell TD, Morris JC, Kukull WA, Centers NIAAsD (2007) The National Alzheimer's Coordinating Center (NACC) database: the Uniform Data Set. Alzheimer Dis Assoc Disord 21: 249-258. 10.1097/ WAD.0b013e318142774e. [PubMed: 17804958]

[32]. Morris JC, Weintraub S, Chui HC, Cummings J, Decarli C, Ferris S, Foster NL, Galasko D, Graff-Radford N, Peskind ER, Beekly D, Ramos EM, Kukull WA (2006) The Uniform Data Set (UDS): clinical and cognitive variables and descriptive data from Alzheimer Disease Centers. Alzheimer Dis Assoc Disord 20: 210-216. 10.1097/01.wad.0000213865.09806.92. [PubMed: 17132964]

[33]. Winblad B, Palmer K, Kivipelto M, Jelic V, Fratiglioni L, Wahlund LO, Nordberg A, Backman L, Albert M, Almkvist O, Arai H, Basun H, Blennow K, de Leon M, DeCarli C, Erkinjuntti T, Giacobini E, Graff C, Hardy J, Jack C, Jorm A, Ritchie K, van Duijn C, Visser P, Petersen RC (2004) Mild cognitive impairment--beyond controversies, towards a consensus: report of the International Working Group on Mild Cognitive Impairment. J Intern Med 256: 240-246. 10.1111/j.1365-2796.2004.01380.x. [PubMed: 15324367]

[34]. McKhann GM, Drachman D, Folstein MF, Katzman R, Price D, Stadlan EM (1984) Clinical diagnosis of Alzheimer's disease: Report of the NINCDS-ADRDA work group. Neurology 34: 939-944. [PubMed: 6610841]

[35]. Cummings JL (1997) The Neuropsychiatric Inventory: Assessing psychopathology in dementia patients. Neurology 48: S10-16.

[36]. Aalten P, de Vugt ME, Lousberg R, Korten E, Jaspers N, Senden B, Jolles J, Verhey FR (2003) Behavioral problems in dementia: a factor analysis of the neuropsychiatric inventory. Dement Geriatr Cogn Disord 15: 99-105. 67972. [PubMed: 12566599]

[37]. Yesavage JA, Brink TL, Rose TL, Lum O, Huang V, Adey M, Leirer VO (1983) Development and validation of a geriatric depression screening scale: A preliminary report. J Psychiatr Res 17: 37-49. 10.1016/0022-3956(82)90033-4.

[38]. Liang KY, Zeger SL (1986) Longitudinal data analysis using generalized linear models. Biometrika 73: 13-22.

[39]. Halekoh U, Højsgaard S, Yan J (2006) The R package geepack for generalized estimating equations. Journal of Statistical Software 15: 1-11.

[40]. R Core Team (2014) R: A language and environment for statistical computing. Vienna, Austria: R Foundation for Statistical Computing.

[41]. Box G, Tidwell P (1962) Transformation of the independent variables. Technometrics 4: 531550.

[42]. Hansen RA, Dusetzina SB, Dominik RC, Gaynes BN (2010) Prescription refill records as a screening tool to identify antidepressant non-adherence. Pharmacoepidemiol Drug Saf 19: 33-37. 10.1002/pds.1881. [PubMed: 19998397]

[43]. Cuijpers P, Berking M, Andersson G, Quigley L, Kleiboer A, Dobson KS (2013) A meta-analysis of cognitive-behavioural therapy for adult depression, alone and in comparison with other treatments. Can J Psychiatry 58: 376-385. 10.1177/070674371305800702. [PubMed: 23870719] 
[44]. Khan A, Faucett J, Lichtenberg P, Kirsch I, Brown WA (2012) A systematic review of comparative efficacy of treatments and controls for depression. PLoS One 7: e41778 10.1371/ journal.pone.0041778. [PubMed: 22860015]

[45]. Rimer J, Dwan K, Lawlor DA, Greig CA, McMurdo M, Morley W, Mead GE (2012) Exercise for depression. Cochrane Database Syst Rev: CD004366. 10.1002/14651858.CD004366.pub5.

[46]. Erickson KI, Voss MW, Prakash RS, Basak C, Szabo A, Chaddock L, Kim JS, Heo S, Alves H, White SM, Wojcicki TR, Mailey E, Vieira VJ, Martin SA, Pence BD, Woods JA, McAuley E, Kramer AF (2011) Exercise training increases size of hippocampus and improves memory. Proc Natl Acad Sci U S A. 10.1073/pnas.1015950108.

[47]. Canevelli M, Grande G, Lacorte E, Quarchioni E, Cesari M, Mariani C, Bruno G, Vanacore N (2016) Spontaneous Reversion of Mild Cognitive Impairment to Normal Cognition: A Systematic Review of Literature and Meta-Analysis. J Am Med Dir Assoc 17: 943-948. 10.1016/ j.jamda.2016.06.020. [PubMed: 27502450]

[48]. Lopez OL, Becker JT, Sweet RA, Klunk W, Kaufer DI, Saxton J, Habeych M, Dekosky ST (2003) Psychiatric symptoms vary with the severity of dementia in probable Alzheimer's disease. The Journal of Neuropsychiatry and Clinical Neurosciences 15: 346-353. 10.1176/jnp.15.3.346. [PubMed: 12928511]

[49]. Leicht H, Berwig M, Gertz HJ (2010) Anosognosia in Alzheimer's disease: the role of impairment levels in assessment of insight across domains. J Int Neuropsychol Soc 16: 463-473. 10.1017/S1355617710000056. [PubMed: 20188013]

[50]. Snow AL, Kunik ME, Molinari VA, Orengo CA, Doody R, Graham DP, Norris MP (2005) Accuracy of self-reported depression in persons with dementia. J Am Geriatr Soc 53: 389-396. 10.1111/j.1532-5415.2005.53154.x. [PubMed: 15743279]

[51]. Bruce JM, Bhalla R, Westervelt HJ, Davis J, Williams V, Tremont G (2008) Neuropsychological correlates of self-reported depression and self-reported cognition among patients with mild cognitive impairment. J Geriatr Psychiatry Neurol 21: 34-40. 10.1177/0891988707311032. [PubMed: 18287168]

[52]. Billioti de Gage S, Begaud B, Bazin F, Verdoux H, Dartigues JF, Peres K, Kurth T, Pariente A (2012) Benzodiazepine use and risk of dementia: prospective population based study. BMJ 345: e6231 10.1136/bmj.e6231. [PubMed: 23045258] 


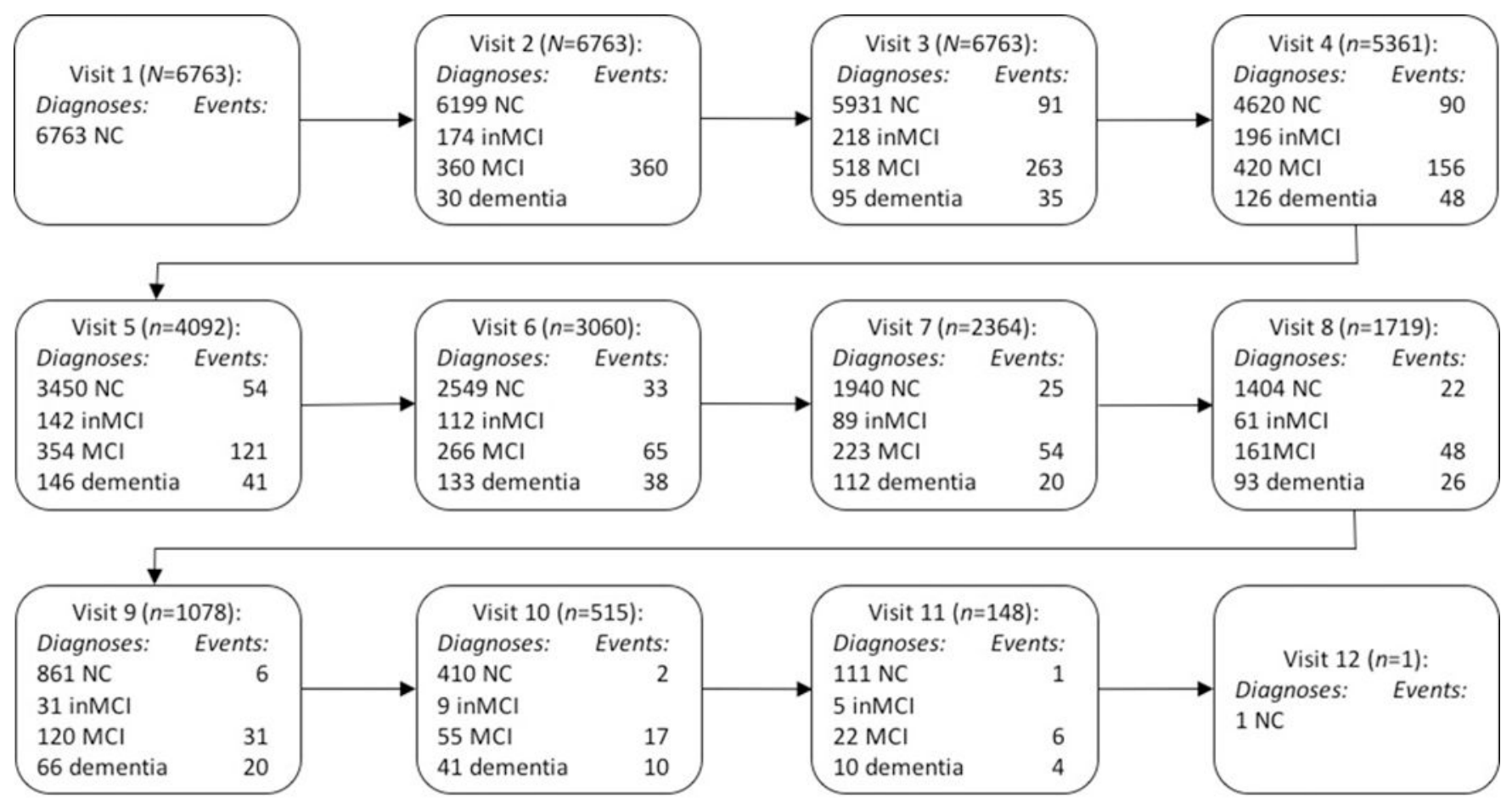

Figure 1.

Flowchart documenting study diagnoses for all participants who met inclusion criteria at all study visits. Visits occurred on an approximately annual basis. $\mathrm{NC}=$ normal cognition; $\mathrm{MCI}$ = mild cognitive impairment; inMCI = cognitively impaired, not MCI. All visits where individuals had a diagnosis of inMCI was excluded from analyses. "Events" documents the number of individuals at each study visit who converted from NC to MCI, reverted from MCI to NC, or progressed from MCI to dementia. Only individuals with dementia due to suspected Alzheimer's disease were included in analyses 
Table 1.

List of medications included in study analyses.

\begin{tabular}{llll}
\hline Antidepressants & Anticonvulsants & Benzodiazepines & Cognition-Enhancing \\
\hline Bupropion & Carbamazepine & Alprazolam & Donepezil \\
Citalopram & Depakote & Clonazepam & Galantamine \\
Duloxetine & Divalproex & Diazepam & Memantine \\
Escitalopram & Eslicarbazepine & Lorazepam & Rivastigmine \\
Fluoxetine & Lamotrigine & & \\
Imipramine & Levetiracetam & & \\
Paroxetine & Oxcarbazepine & & \\
Sertraline & Phenytoin & & \\
Venlafaxine & Pregabalin & & \\
& Topiramate & & \\
\hline
\end{tabular}


Table 2.

Demographic characteristics of the entire sample as well as the subsets of participants that experienced the three critical events. For individual who progressed to AD dementia, only those who had a presumptive etiology of possible or probable Alzheimer's disease (AD) were included in analyses. MCI = Mild cognitive impairment, $\mathrm{NC}=$ Normal cognition, $\mathrm{W}=$ White, $\mathrm{AA}=$ African American.

\begin{tabular}{lccccc}
\hline & Total Sample & NC all visits & Convert to MCI & Revert to NC & Progress to AD \\
\cline { 2 - 6 }$n$ & 6763 & 5173 & 1121 & 324 & 242 \\
Baseline age (SD) & $72.3(9.1)$ & $71.0(8.8)$ & $77.0(8.6)$ & $74.4(8.8)$ & $79.9(7.8)$ \\
Sex (\% female) & $66.3 \%$ & $67.7 \%$ & $60.9 \%$ & $59.6 \%$ & $67.8 \%$ \\
Education (SD) & $15.7(3.0)$ & $15.9(3.0)$ & $15.3(3.2)$ & $15.5(3.2)$ & $15.3(3.0)$ \\
& $81 \% \mathrm{~W}, 13 \% \mathrm{AA}$ & $81 \% \mathrm{~W}, 14 \% \mathrm{AA}$, & $82 \% \mathrm{~W}, 13 \% \mathrm{AA}$ & $78 \% \mathrm{~W}, 18 \% \mathrm{AA}$ & $87 \% \mathrm{~W}, 10 \% \mathrm{AA}$ \\
Race & $5 \%$ other & $5 \%$ other & $5 \%$ other & $4 \%$ other & $4 \%$ other \\
Yrs since baseline at event (SD) & $\mathrm{NA}$ & $\mathrm{NA}$ & $3.4(2.3)$ & $4.3(2.0)$ & $5.5(2.2)$ \\
Follow-up visits at event (SD) & $\mathrm{NA}$ & $\mathrm{NA}$ & $2.9(2.1)$ & $3.7(1.7)$ & $4.8(2.1)$ \\
Total follow-up length (SD) & $5.4(2.5)$ & $5.3(2.5)$ & $6.2(2.3)$ & $6.6(2.3)$ & $6.8(2.2)$ \\
Total follow-up visits (SD) & $4.8(2.3)$ & $4.6(2.3)$ & $6.0(2.5)$ & $5.9(2.2)$ & $6.0(2.2)$ \\
\hline
\end{tabular}


Table 3.

Results from generalized linear models estimated by generalized estimating equations predicting the conversion from NC to MCI compared to remaining stable at NC. Positive coefficients indicate an increased probability of conversion to MCI at higher levels of the variable. All predictors in Models 1B-E were added to the baseline predictors in Model 1A. GDS-15 = Geriatric Depression Scale. Hyperactivity, Psychosis, Mood, and Anxiety symptoms were derived from the Neuropsychiatric Inventory Questionnaire. Both the total score at each study visit (Models 1B and 1C) and the change in symptoms between study visits (1D and 1E) were analyzed in models. "Years" represents the amount of time since the initial study visit. "Age" refers to age at the baseline visit.

\begin{tabular}{|c|c|c|c|c|c|}
\hline \multirow[b]{2}{*}{ Effect } & \multicolumn{4}{|c|}{ Analysis 1: Conversion from NC to $\mathrm{MCI}$} & \multirow[b]{2}{*}{$\operatorname{Exp}(B)$} \\
\hline & B & 95\% CI & Wald Z & $p$ & \\
\hline \multicolumn{6}{|l|}{ Model $1 A$} \\
\hline Intercept & -6.95 & {$[-7.72,-6.19]$} & 314.90 & $<.001$ & $<0.01$ \\
\hline Years & -0.00 & {$[-0.03,0.03]$} & 0.00 & .970 & 1.00 \\
\hline Age & 0.07 & {$[0.06,0.08]$} & 303.50 & $<.001$ & 1.07 \\
\hline $\operatorname{Sex}$ & -0.28 & {$[-0.41,-0.15]$} & 18.40 & $<.001$ & 0.75 \\
\hline Education & -0.04 & {$[-0.06,-0.02]$} & 16.20 & $<.001$ & 0.96 \\
\hline \multicolumn{6}{|l|}{ Model $1 B$} \\
\hline GDS-15 total & 0.17 & {$[0.15,0.20]$} & 180.73 & $<.001$ & 1.19 \\
\hline \multicolumn{6}{|l|}{ Model $1 C$} \\
\hline Hyperactivity & 0.18 & {$[0.11,0.25]$} & 28.91 & $<.001$ & 1.20 \\
\hline Psychosis & 0.23 & {$[-0.03,0.49]$} & 3.08 & .079 & 1.26 \\
\hline Mood & 0.17 & {$[0.12,0.23]$} & 40.29 & $<.001$ & 1.19 \\
\hline Anxiety & 0.25 & {$[0.12,0.39]$} & 13.32 & $<.001$ & 1.29 \\
\hline \multicolumn{6}{|l|}{ Model $1 D$} \\
\hline GDS-15 change & 0.05 & {$[0.00,0.09]$} & 4.35 & .037 & 1.05 \\
\hline \multicolumn{6}{|l|}{ Model $1 E$} \\
\hline Hyperactivity change & 0.14 & {$[0.04,0.24]$} & 7.92 & .005 & 1.15 \\
\hline Psychosis change & 0.27 & {$[-0.12,0.67]$} & 1.82 & .177 & 1.31 \\
\hline Mood change & 0.08 & {$[0.01,0.15]$} & 5.04 & .025 & 1.09 \\
\hline Anxiety change & 0.15 & {$[-0.02,0.32]$} & 3.03 & .082 & 1.16 \\
\hline
\end{tabular}




\section{Table 4.}

Results from generalized linear models estimated by generalized estimating equations predicting the reversion from MCI to NC. Negative coefficients indicate an increased probability of reversion to NC at lower levels of the variable. All predictors in Models 2B-E were added to the baseline predictors in Model 2A. GDS-15 = Geriatric Depression Scale. Hyperactivity, Psychosis, Mood, and Anxiety symptoms were derived from the Neuropsychiatric Inventory Questionnaire. aMCI represents the coefficient for the change in likelihood of reversion for participants with amnestic compared to non-amnestic MCI.

\begin{tabular}{|c|c|c|c|c|c|}
\hline \multicolumn{6}{|c|}{ Analysis 2: Reversion to NC } \\
\hline Effect & B & $95 \% \mathrm{CI}$ & Wald Z & $p$ & $\operatorname{Exp}(B)$ \\
\hline \multicolumn{6}{|l|}{ Model $2 A$} \\
\hline Intercept & 2.39 & {$[0.85,3.92]$} & 9.31 & .002 & 10.90 \\
\hline Years & -0.12 & {$[-0.19,-0.06]$} & 15.73 & $<.001$ & 0.88 \\
\hline Age & -0.04 & {$[-0.06,-0.03]$} & 26.22 & $<.001$ & 0.96 \\
\hline Sex & 0.01 & {$[-0.27,0.29]$} & 0.00 & .960 & 1.01 \\
\hline Education & 0.04 & {$[-0.01,0.08]$} & 2.40 & .121 & 1.04 \\
\hline $\mathrm{aMCI}$ & -0.38 & {$[-0.65,0.11]$} & 7.50 & .006 & 0.68 \\
\hline \multicolumn{6}{|l|}{ Model $2 B$} \\
\hline GDS-15 total & -0.09 & {$[-0.15,-0.03]$} & 8.89 & .003 & 0.91 \\
\hline \multicolumn{6}{|l|}{ Model 2C } \\
\hline Hyperactivity & -0.04 & {$[-0.19,0.10]$} & 0.33 & .568 & 0.96 \\
\hline Psychosis & -0.17 & {$[-0.56,0.23]$} & 0.68 & .411 & 0.85 \\
\hline Mood & -0.12 & {$[-0.23,-0.02]$} & 5.14 & .023 & 0.89 \\
\hline Anxiety & -0.36 & {$[-0.60,-0.12]$} & 8.44 & $<.001$ & 0.70 \\
\hline \multicolumn{6}{|l|}{ Model 2D } \\
\hline GDS-15 change & -0.08 & {$[-0.14,-0.01]$} & 5.53 & .019 & 0.93 \\
\hline \multicolumn{6}{|l|}{ Model $2 E$} \\
\hline Hyperactivity change & 0.02 & {$[-0.07,0.11]$} & 0.25 & .616 & 1.02 \\
\hline Psychosis change & -0.15 & {$[-0.39,0.09]$} & 1.43 & .232 & 0.86 \\
\hline Mood change & -0.06 & {$[-0.13,0.02]$} & 2.32 & .128 & 0.95 \\
\hline Anxiety change & -0.07 & {$[-0.23,0.08]$} & 0.83 & .361 & 0.93 \\
\hline
\end{tabular}


Table 5.

Results from generalized linear models estimated by generalized estimating equations predicting the progression from MCI to Alzheimer's disease (AD) dementia. Positive coefficients indicate an increased probability of progression to AD dementia at higher levels of the variable. All predictors in Models 3B-E were added to the baseline predictors in Model 3A. GDS-15 = Geriatric Depression Scale. Hyperactivity, Psychosis, Mood, and Anxiety symptoms were derived from the Neuropsychiatric Inventory Questionnaire.

\begin{tabular}{|c|c|c|c|c|c|}
\hline \multicolumn{6}{|c|}{ Analysis 3: Progression to AD dementia } \\
\hline Effect & B & $95 \% \mathrm{CI}$ & Wald Z & $p$ & $\operatorname{Exp}(B)$ \\
\hline \multicolumn{6}{|l|}{ Model $3 A$} \\
\hline Intercept & -6.01 & {$[-7.93,-4.08]$} & 37.30 & $<.001$ & $<0.01$ \\
\hline Years & 0.09 & {$[0.03,0.16]$} & 7.76 & .005 & 1.10 \\
\hline Age & 0.03 & {$[0.01,0.05]$} & 12.16 & $<.001$ & 1.03 \\
\hline Sex & 0.30 & {$[-0.02,0.62]$} & 3.35 & .067 & 1.35 \\
\hline Education & 0.01 & {$[-0.04,0.06]$} & 0.17 & .684 & 1.01 \\
\hline aMCI & 0.85 & {$[0.37,1.33]$} & 12.02 & .001 & 2.33 \\
\hline \multicolumn{6}{|l|}{ Model $3 B$} \\
\hline GDS-15 total & 0.05 & {$[-0.01,0.12]$} & 3.14 & .076 & 1.06 \\
\hline \multicolumn{6}{|l|}{ Model 3C } \\
\hline Hyperactivity & 0.17 & {$[0.04,0.30]$} & 6.78 & .009 & 1.19 \\
\hline Psychosis & 0.13 & {$[-0.21,0.48]$} & 0.58 & 0.45 & 1.14 \\
\hline Mood & 0.28 & {$[0.18,0.37]$} & 31.89 & $<.001$ & 1.32 \\
\hline Anxiety & -0.03 & {$[-0.30,0.23]$} & 0.07 & .795 & 0.97 \\
\hline \multicolumn{6}{|l|}{ Model 3D } \\
\hline GDS- 15 change & 0.06 & {$[-0.03,0.15]$} & 1.57 & .210 & 1.06 \\
\hline \multicolumn{6}{|l|}{ Model $3 E$} \\
\hline Hyperactivity change & 0.17 & {$[0.03,0.32]$} & 5.47 & .019 & 1.19 \\
\hline Psychosis change & 0.31 & {$[-0.04,0.66]$} & 3.10 & .078 & 1.37 \\
\hline Mood change & 0.14 & {$[0.03,0.25]$} & 6.32 & .012 & 1.15 \\
\hline Anxiety change & -0.01 & {$[-0.29,0.28]$} & 0.00 & .957 & 0.99 \\
\hline
\end{tabular}


Table 6.

Results from generalized linear models estimated by generalized estimating equations predicting the conversion from NC to MCI. All predictors in these models were added to the baseline predictors from Model 1A (Table 3). Positive coefficients indicate that having a prescription for one or more of the medications was associated with a higher probability of converting to MCI. GDS-15 = Geriatric Depression Scale. Hyperactivity, Mood, and Anxiety symptoms were derived from the Neuropsychiatric Inventory Questionnaire.

\begin{tabular}{|c|c|c|c|c|c|}
\hline \multicolumn{6}{|c|}{ Analysis 4: Medications and Conversion to MCI } \\
\hline Effect & B & $95 \% \mathrm{CI}$ & Wald $\mathrm{Z}$ & $p$ & $\operatorname{Exp}(\mathbf{B})$ \\
\hline \multicolumn{6}{|l|}{ Model $4 A$} \\
\hline Antidepressants & 0.46 & {$[0.28,0.64]$} & 24.52 & $<.001$ & 1.58 \\
\hline \multicolumn{6}{|l|}{ Model $4 B$} \\
\hline Antidepressants & 0.07 & {$[-0.14,0.28]$} & 0.40 & .529 & 1.07 \\
\hline GDS-15 total & 0.12 & {$[0.09,0.15]$} & 66.66 & $<.001$ & 1.13 \\
\hline Hyperactivity & 0.20 & {$[0.13,0.26]$} & 34.42 & $<.001$ & 1.22 \\
\hline Mood & 0.12 & {$[0.07,0.18]$} & 18.04 & $<.001$ & 1.13 \\
\hline Anxiety & 0.23 & {$[0.09,0.36]$} & 10.26 & .001 & 1.25 \\
\hline \multicolumn{6}{|l|}{ Model 4C } \\
\hline Anticonvulsants & 0.53 & {$[0.02,1.04]$} & 4.20 & .040 & 1.70 \\
\hline \multicolumn{6}{|l|}{ Model 4D } \\
\hline Anticonvulsants & 0.43 & {$[-0.09,0.95]$} & 2.58 & .108 & 1.53 \\
\hline GDS-15 total & 0.12 & {$[0.09,0.15]$} & 70.34 & $<.001$ & 1.13 \\
\hline Hyperactivity & 0.20 & {$[0.13,0.26]$} & 34.57 & $<.001$ & 1.22 \\
\hline Mood & 0.13 & {$[0.07,0.18]$} & 18.59 & $<.001$ & 1.13 \\
\hline Anxiety & 0.23 & {$[0.09,0.37]$} & 10.45 & .001 & 1.26 \\
\hline \multicolumn{6}{|l|}{ Model $4 E$} \\
\hline Benzodiazepines & 0.24 & {$[-0.03,0.51]$} & 3.09 & .079 & 1.27 \\
\hline \multicolumn{6}{|l|}{ Model $4 F$} \\
\hline Cognition Meds & 1.76 & {$[0.94,2.57]$} & 17.8 & $<.001$ & 5.79 \\
\hline \multicolumn{6}{|l|}{ Model $4 G$} \\
\hline Cognition Meds & 1.34 & {$[0.42,2.26]$} & 8.16 & .004 & 3.83 \\
\hline GDS-15 total & 0.12 & {$[0.09,0.15]$} & 68.77 & $<.001$ & 1.13 \\
\hline Hyperactivity & 0.20 & {$[0.13,0.26]$} & 34.49 & $<.001$ & 1.22 \\
\hline Mood & 0.13 & {$[0.07,0.18]$} & 18.65 & $<.001$ & 1.13 \\
\hline Anxiety & 0.23 & {$[0.09,0.36]$} & 10.31 & .001 & 1.25 \\
\hline
\end{tabular}


Table 7.

Results from generalized linear models estimated by generalized estimating equations predicting reversion from MCI to NC. All predictors in these models were added to the baseline predictors from Model 2A (Table 4). Models include the presence/absence of various medication classes, as well as whether participants received a new prescription for an antidepressant or a cognition-enhancing medication since the previous study visit. Negative coefficients indicate that individuals with a prescription for one or more of the medications had a decreased probability of reverting to NC.

\begin{tabular}{cccccc}
\hline \multicolumn{7}{c}{ Analysis 5: Medications and Reversion to NC } \\
Effect & B & $\mathbf{9 5 \%}$ CI & Wald Z & $p$ & Exp(B) \\
\hline Model 5A & & & & & \\
Antidepressants & -0.11 & {$[-0.46,0.24]$} & 0.37 & .544 & 0.90 \\
Model 5B & & & & & \\
New antidep. & -0.13 & {$[-0.81,0.54]$} & 0.15 & .700 & 0.87 \\
Model 5C & & & & & \\
Benzodiazepines & -0.56 & {$[-1.20,0.09]$} & 2.88 & .090 & 0.57 \\
Model 5D & & & & & \\
Cognition Meds & $-\mathbf{1 . 5 1}$ & {$[-\mathbf{2 . 6 3 , 0 . 4 0 ]}$} & $\mathbf{7 . 0 5}$ & .008 & $\mathbf{0 . 2 2}$ \\
Model 5E & & & & & \\
New Cog. Med & $-\mathbf{1 . 3 0}$ & {$[-\mathbf{2 . 3 7 , - 0 . 2 3 ]}$} & $\mathbf{5 . 6 4}$ & .018 & $\mathbf{0 . 2 7}$ \\
\hline
\end{tabular}


Table 8.

Results from generalized linear models estimated by generalized estimating equations predicting the progression from MCI to Alzheimer's disease (AD) dementia. All predictors in these models were added to the baseline predictors from Model 3A (Table 5). Hyperactivity and Mood symptoms were derived from the Neuropsychiatric Inventory Questionnaire. Models include the presence/absence of various medication classes, as well as whether participants received a new prescription for a cognition-enhancing medication since the previous study visit. Positive coefficients indicate that having a prescription for one or more of the medications was associated with a higher probability of progressing to AD dementia.

\begin{tabular}{|c|c|c|c|c|c|}
\hline \multicolumn{6}{|c|}{ Analysis 6: Medications and Progression to AD dementia } \\
\hline Effect & $\mathbf{B}$ & $95 \% \mathrm{CI}$ & Wald $\mathrm{Z}$ & $p$ & $\operatorname{Exp}(B)$ \\
\hline \multicolumn{6}{|l|}{ Model $6 A$} \\
\hline Antidepressants & 0.69 & {$[0.36,1.03]$} & 16.33 & $<.001$ & 2.00 \\
\hline \multicolumn{6}{|l|}{ Model $6 B$} \\
\hline Antidepressants & 0.48 & {$[0.11,0.85]$} & 6.50 & .011 & 1.62 \\
\hline Hyperactivity & 0.20 & {$[0.08,0.32]$} & 10.70 & .001 & 1.22 \\
\hline Mood & 0.25 & {$[0.16,0.34]$} & 27.87 & $<.001$ & 1.28 \\
\hline \multicolumn{6}{|l|}{ Model 6C } \\
\hline Benzodiazepines & -0.16 & {$[-0.85,0.53]$} & 0.21 & .648 & 0.85 \\
\hline \multicolumn{6}{|l|}{ Model $6 D$} \\
\hline Cognition Meds & 1.01 & {$[0.41,1.62]$} & 10.73 & .001 & 2.75 \\
\hline \multicolumn{6}{|l|}{ Model $6 E$} \\
\hline Cognition Meds & 0.98 & {$[0.26,1.70]$} & 7.06 & .008 & 2.67 \\
\hline Hyperactivity & 0.20 & {$[0.07,0.32]$} & 9.75 & .002 & 1.22 \\
\hline Mood & 0.26 & {$[0.17,0.35]$} & 31.27 & $<.001$ & 1.30 \\
\hline \multicolumn{6}{|l|}{ Model $6 F$} \\
\hline New Cog. Med & 1.11 & {$[0.46,1.76]$} & 11.16 & .001 & 3.03 \\
\hline \multicolumn{6}{|l|}{ Model $6 G$} \\
\hline New Cog. Med & 1.15 & {$[0.42,1.88]$} & 9.62 & .002 & 3.17 \\
\hline Hyperactivity & 0.20 & {$[0.08,0.32]$} & 9.49 & .002 & 1.22 \\
\hline Mood & 0.26 & {$[0.16,0.36]$} & 32.23 & $<.001$ & 1.30 \\
\hline
\end{tabular}

